\title{
Assessment of in vivo bond strength studies of the orthodontic bracket-adhesive system: A systematic review
}

\author{
Tamzid Ahmed ${ }^{1}$, Norma Ab Rahman ${ }^{1}$, Mohammad Khursheed Alam²
}

Correspondence: Dr. Norma Ab Rahman

Email:drnorma@usm.my

\author{
1Orthodontics Unit, School of Dental \\ Sciences (PPSG), Universiti Sains Malaysia, \\ Kota Bharu, Kelantan, Malaysia, \\ 2Department of Orthodontics, College of Dentistry, \\ Jouf University, Sakaka, Kingdom of Saudi Arabia
}

\section{ABSTRACT}

The aim of this study was to systematically review the available studies measuring the bond strength of orthodontic bracket-adhesive system under different experimental conditions in vivo. Literature search was performed in four different databases: PubMed, Web of Science, Cochrane, and Scopus using the keywords - bond strength, orthodontic brackets, bracket-adhesive, and in vivo. A total of six full-text articles were selected based on the inclusion and exclusion criteria of our study after a careful assessment by the two independent reviewers. Data selection was performed by following PRISMA 2009 guidelines. Five of the selected studies were clinical trials; one study was a randomized clinical trial. From each of the selected articles, the following data were extracted - number of samples, with the type of tooth involved materials under experiment methods of measurement, the time interval between bonding and debonding orthodontic brackets, mode of force application, and the bond strength results with the overall outcome. The methodological quality assessment of each article was done by the modified Downs and Black checklist method. The qualitative analyses were done by two independent reviewers. Conflicting issues were resolved in a consensus meeting by consulting the third reviewer (MKA). Meta-analysis could not be performed due to the lack of homogenous study results. The review reached no real conclusion apart from the lack of efforts to clinically evaluate the bonding efficiency of a wide range of orthodontic bracket-adhesive systems in terms of debonding force compared to laboratory-based in vitro and ex vivo studies.

Key words: Bond strength, bracket-adhesive, in vivo, orthodontic brackets

\section{INTRODUCTION}

The efficacy of laborious and prolonged-fixed orthodontic treatment requires a stable bonding between the brackets and the teeth surface. Many materials as orthodontic adhesives, surface preparation techniques, and bonding methods have been experimented by the researchers for the advancement of the bracket bonding system. The potency of such bonding system is usually tested in a laboratory using

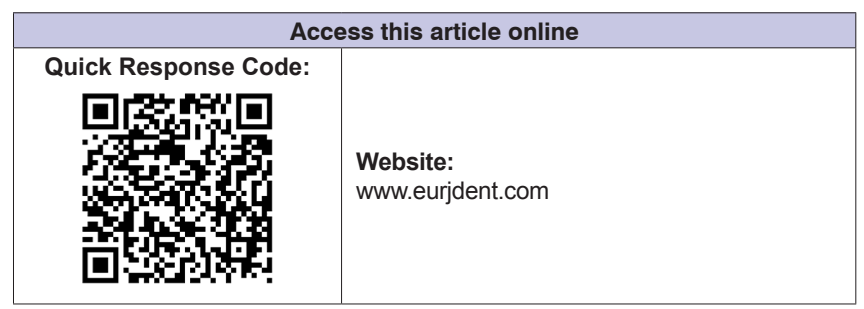

a mechanical testing machine. The disadvantage of such testing method is that the effect of aging and biodegradation of the bonding material in the oral environment is omitted. Therefore, the test results do not seem to have a correlation with the clinical failure rates of the brackets which are reported as high as $0.5 \%-55.8 \%$ by many studies (Linklater and Gordon 2001 and Hobson et al. 2001). Hence, it is justifiable to

This is an open access journal, and articles are distributed under the terms of the Creative Commons Attribution-NonCommercial-ShareAlike 4.0 License, which allows others to remix, tweak, and build upon the work non-commercially, as long as appropriate credit is given and the new creations are licensed under the identical terms.

For reprints contact: reprints@medknow.com

How to cite this article: Ahmed T, Rahman NA, Alam MK. Assessment of in vivo bond strength studies of the orthodontic bracket-adhesive system: A systematic review. Eur J Dent 2018;12:602-9.

DOI: 10.4103/ejd.ejd_22_18 
say that in vivo bond strength tests are more precise to experiment the efficacy of orthodontic bonding materials. Few researchers innovated the way to measure the bond strength of orthodontic brackets inside the oral environment with a custom-made instrument equipped with a measuring device.

The main PICO question of this study is: how are the accuracy, reliability, and the efficiency of different techniques measuring the bond strength of orthodontic brackets with the influence of variables in vivo? and what are the potential research gaps in comparison to the in vitro and ex vivo studies?

Therefore, the aim of this study is to systematically review the in vivo clinical studies available in the literature measuring the debonding force of orthodontic brackets to find the answer to our (PICO) question by assessing and comparing the accuracy and reliability of different methods, measurement techniques, and the influence of variable parameters on the study results.

\section{MATERIALS AND METHODS}

The review is not registered under any organization. The review was performed by following the Preferred Reporting Items for Systemic Reviews and Meta-Analyses (PRISMA) 2009 statements (http://www.prisma-statement.org). An independent literature search was made by two review authors (TA and NAR) in four different databases: PubMed (last date of search February 18, 2017), Web of Science (last date of search February 21, 2017), Scopus (last date of search February 23, 2017), and Cochrane (last date of search February 23, 2017) using the keywords -bond strength, Orthodontic Brackets, Bracket-Adhesive, and Invivo. There was no language restriction for the search. In addition, hand searching was also performed in the library for any relevant articles. Initially, the titles and abstracts of the resultant articles relevant to our study topic: the bond strength of orthodontic brackets in vivo were analyzed by two review authors (TA and NAR), independently. The references of the resultant studies were also searched for any related studies missed during the initial search. The search strategy of the database is presented in Table 1. The following criteria were constructed a priori to select the articles for inclusion in this review. The inclusion criteria of this review consist of (1) in vivo clinical trials and case-control studies that debonded and measured the bond strength of orthodontic brackets with a suitable experimental device, (2) all age and sex, (3) all languages, and (4) no sample size restrictions. The exclusion criteria were as follows: (1) longitudinal clinical studies or clinical trials of in vivo bracket-failure rates and survival rates, ex vivo clinical trials where the orthodontic brackets were debonded and the bond strength measured extraorally after temporarily exposing them to the oral environment, (2) any conference abstracts and case reports, and (3) editorials and opinions. The titles and abstracts of all the included studies were downloaded in Endnote software version X7 (Thomson, Reuters, Carlsbad, California, USA) cross-referenced and the duplicate studies were excluded after the careful assessment and comparison of the study titles, author names, sample sizes, interventions, and the outcomes by one reviewer (TA). The full-text articles were assessed by the two independent reviewers (TA and NAR) for eligibility. The quality assessment (risk of bias) of the selected studies was also assessed by the same reviewers (TA and NAR) according to the modified Downs and Black checklist. ${ }^{[1]}$ The criteria for assessing each study were grouped into five principal segments: reporting, external validity, internal validity-bias, internal validity-confounding, and power. Items were given 1 point if the respective criteria were fulfilled. Missing or unable to determine any criteria was assessed by giving 0 points. In the power domain, instead of assessing the power of the studies to detect the clinically important effect on the scale of 1-5 points, the studies that determined sample size using power were given 1 point and 0 points if not. From the total of 27 points, the articles that scored $<17$ points were judged with the presence of methodological limitation. The following data were extracted from each study included: the sample size with the number and type of teeth involved, materials used, type of instrument and measuring device used, the mode and the location of force application, the duration of exposure of the brackets

\begin{tabular}{lllcl}
\multicolumn{2}{l}{ Table 1: Search strategy for database } & & \\
\hline Name of database & Last date of search & Keywords & Study results & Study selected \\
\hline PubMed & January 15, 2018 & Bond strength, & 47 & 20 \\
Web of Science & January 15, 2018 & orthodontic brackets, & 99 & 31 \\
Scopus & January 15, 2018 & bracket-adhesive, In vivo & 50 & 22 \\
Cochrane & January 15, 2018 & & 22 & 10 \\
\hline
\end{tabular}


to the oral environment before debonding, and the bond strength results with the overall outcome. Again, the data were extracted by two independent reviewers (TA and NAR). Resolution of any disagreement was done in a consensus meeting; the consult was also taken from the third reviewer (MKA). The whole process of study selection, data extraction, and the risk of bias assessment was performed unblinded.

\section{RESULTS}

The number of studies found by searching through different databases and the selection procedure is detailed in the flow diagram according to PRISMA 2009 guidelines, which is presented below in Figure 1. From the total of 209 found articles from the databases, 38 abstracts were retrieved and at last eight full-text articles were assessed and analyzed. Two articles were rejected because they did not apply their instrument to measure the in vivo bond strength of orthodontic brackets. The number of excluded articles with the reasons is presented in Table 2. Additional papers could not be found through hand searching. Among the six included studies, five of them were clinical trials ${ }^{[2-6]}$ and only one study was a randomized clinical trial. ${ }^{[7]}$ All the articles were published in English, except for one in Portuguese. ${ }^{[5]}$ The Portuguese article was translated into English with the help of "Google Translator" by TA. The quality assessment result of each included studies according to modified Downs and Black checklist. ${ }^{[1]}$ it is presented in an additional file. According to the assessment, 5 of the studies scored 17 and above and rated as high-quality, ${ }^{[2-4,6,7]}$ only one study scored 10 which is rated as lower quality. ${ }^{[5]}$ The extracted data from each of the study are summarized in Table 3. No studies found to estimate the sample size using the power of the study.

\section{PRISMA 2009 Flow Diagram}

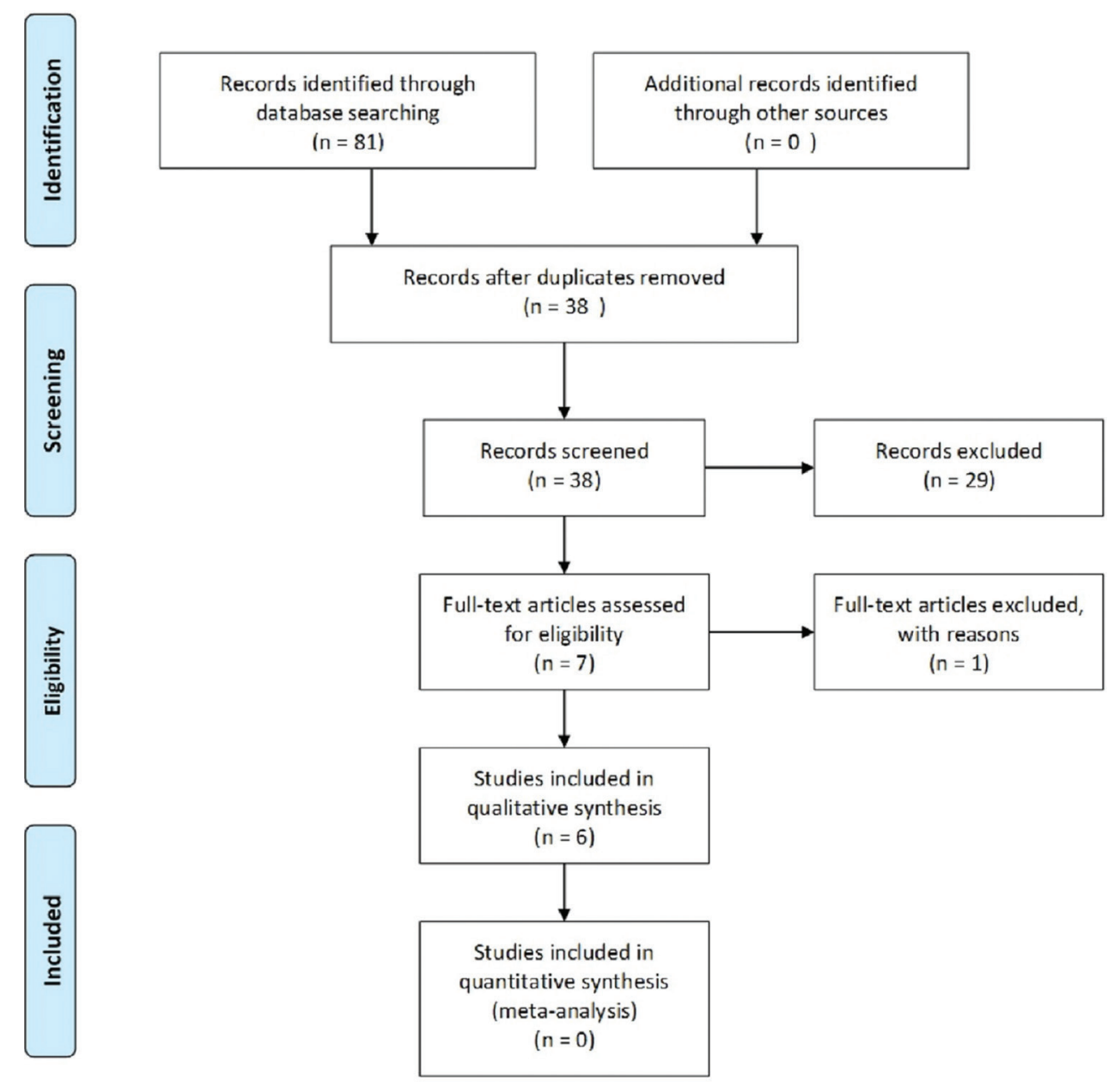

Figure 1: Preferred reporting items for systemic reviews and meta-analyses flow diagram of the study selection 


\begin{tabular}{|c|c|}
\hline Study & Reason for exclusion \\
\hline Tonus et al., 2007 & In vivo bond strength not measured \\
\hline Varlika et al., 2009 & In vivo bond failure rate \\
\hline Uysal et al., 2010 & $\begin{array}{l}\text { In vivo demineralization of enamel } \\
\text { around orthodontic brackets }\end{array}$ \\
\hline Uysal et al., 2010 & $\begin{array}{l}\text { In vivo enamel demineralization } \\
\text { around orthodontic brackets }\end{array}$ \\
\hline Uysal et al., 2010 & $\begin{array}{l}\text { In vivo enamel demineralization } \\
\text { around orthodontic brackets }\end{array}$ \\
\hline Summers et al., 2004 & $\begin{array}{l}\text { Bond strength measured } \\
\text { on extracted teeth }\end{array}$ \\
\hline Signorelli et al., 2006 & In vivo bracket failure rate \\
\hline Shammaa et al., 1999 & In vivo bracket survival rate \\
\hline Rosenbach et al., 2007 & $\begin{array}{l}\text { Bond strength measured } \\
\text { on extracted teeth }\end{array}$ \\
\hline Prietsch et al., 2007 & In vivo bond strength not measured \\
\hline Praxedes-Neto et al., 2012 & $\begin{array}{l}\text { In vivo remineralization } \\
\text { of acid-etch enamel }\end{array}$ \\
\hline Polat et al., 2004 & In vivo bracket failure rate \\
\hline Penido et al., 2009 & $\begin{array}{l}\text { The full-text article could } \\
\text { not be retrieved }\end{array}$ \\
\hline Pasquale et al., 2007 & In vivo bond failure rate \\
\hline Pascotto et al., 2004 & $\begin{array}{l}\text { In vivo enamel demineralization } \\
\text { around orthodontic brackets }\end{array}$ \\
\hline Ozer et al., 2005 & In vivo bond failure rate \\
\hline Örtendahl 1998 & Ex vivo study \\
\hline Nirupama et al., 2012 & $\begin{array}{l}\text { Bond strength measured } \\
\text { on extracted teeth }\end{array}$ \\
\hline Murray et al., 2003 & $\begin{array}{l}\text { Bond strength measured } \\
\text { on extracted teeth }\end{array}$ \\
\hline Mullins et al., 2009 & In vivo bracket failure rate \\
\hline Mirzakouchaki et al., 2016 & $\begin{array}{l}\text { Bond strength measured } \\
\text { on extracted teeth }\end{array}$ \\
\hline Linklater et al., 2003 & In vivo bond failure rate \\
\hline Le et al., 2003 & $\begin{array}{l}\text { In vivo enamel demineralization } \\
\text { around orthodontic brackets }\end{array}$ \\
\hline Krishnaswamy et al., 2007 & In vivo bond failure rate \\
\hline Korbmacher et al., 2006 & In vitro bond strength measurement \\
\hline Ireland et al., 2003 & In vivo bond failure rate \\
\hline Hammad et al., 2013 & $\begin{array}{l}\text { Bond strength measured } \\
\text { on extracted teeth }\end{array}$ \\
\hline Grover et al., 2012 & In vivo bond failure rate \\
\hline Ghiz et al., 2009 & $\begin{array}{l}\text { In vivo enamel demineralization } \\
\text { around orthodontic brackets }\end{array}$ \\
\hline de Moura et al., 2006 & $\begin{array}{l}\text { In vivo enamel demineralization } \\
\text { around orthodontic brackets }\end{array}$ \\
\hline Chatzistavrou et al., 2009 & $\begin{array}{l}\text { Bond strength measured } \\
\text { on extracted teeth }\end{array}$ \\
\hline Cal-Neto et al., 2006 & $\begin{array}{l}\text { Bond strength measured on } \\
\text { extracted teeth }\end{array}$ \\
\hline
\end{tabular}

Premolar teeth were predominantly used as a sample to measure the bond strength. For enamel surface preparation before bonding orthodontic brackets, self-etching primer, and $37 \%$ phosphoric acid was used predominantly. ${ }^{[2,5-7]}$ Two studies did not mention the enamel surface preparation technique. ${ }^{[3,4]}$ In vivo polymerization of the orthodontic adhesives was done by the visible light for $40 \mathrm{~s}$ by two studies, while another study attempted polymerization using halogen light for $20 \mathrm{~s} \cdot{ }^{[2,5,7]}$ One study compared the bond strength of orthodontic brackets in two groups after curing the bonding material with the argon laser for $10 \mathrm{~s}$ and conventional visible light for $40 \mathrm{~s}$, respectively, and found no difference. ${ }^{[7]}$ All the included studies characterize the bond strength as average stress in megapascal $(\mathrm{MPa})$ units after dividing the debonding force in newtons $(\mathrm{N})$ by the bracket surface area in square millimeters $\left(\mathrm{mm}^{2}\right)$. The most common mode of force application was shear/peel loading on the area between the bracket wings and the base among all the selected studies. One study emphasized the influence of force location on the bond strength of orthodontic brackets. ${ }^{[3]}$ After 10 min of bonding, the time duration between the bonding and debonding has no influence on the bond strength in vivo. In comparison to the in vitro bond strength values, the in vivo bond strength of the orthodontic brackets found lower in two studies following the similar method of measurement. ${ }^{[2,4]} \mathrm{A}$ custom-made debonding instrument equipped with digital force gauge was mostly used for debonding force measurement in vivo. ${ }^{[2,4-6]}$ Two studies applied strain gauge to measure the bracket debonding force. . $^{[3,7]}$ Commercially manufactured regular debonding plier was used in only one study. ${ }^{[3]}$

\section{DISCUSSION}

To minimize the possibility of missing any potential study relevant to our search, the inclusion criteria were lenient. There were no restrictions to the publication year, the search was not confined to specific language, limitations were not also applied for the sample size. Despite all the measures, the number of studies eligible for inclusion is only six. Lack of regular turnover of the patients receiving fixed orthodontic therapy, dimension, and the limitation of the instruments with a measuring device in terms of access, safety, and efficacy may be the attributing factors that the researchers are more focused on laboratory-based bond strength measurement. Studies that are titled as the in vivo bond strength of orthodontic brackets, but actually measured the bond strength on the teeth that are already planned for extraction after exposing them to the oral environment for certain period are excluded. In these cases, the brackets and the adhesives may get exposure to the oral environment, but these are practically ex vivo studies because the bond strength is measured outside preferably in a laboratory by a mechanical testing device. Transbond XT (3M Unitek, Monrovia, California, USA) light-cured composite 
Ahmed, et al.: Bond strength of orthodontic brackets in vivo

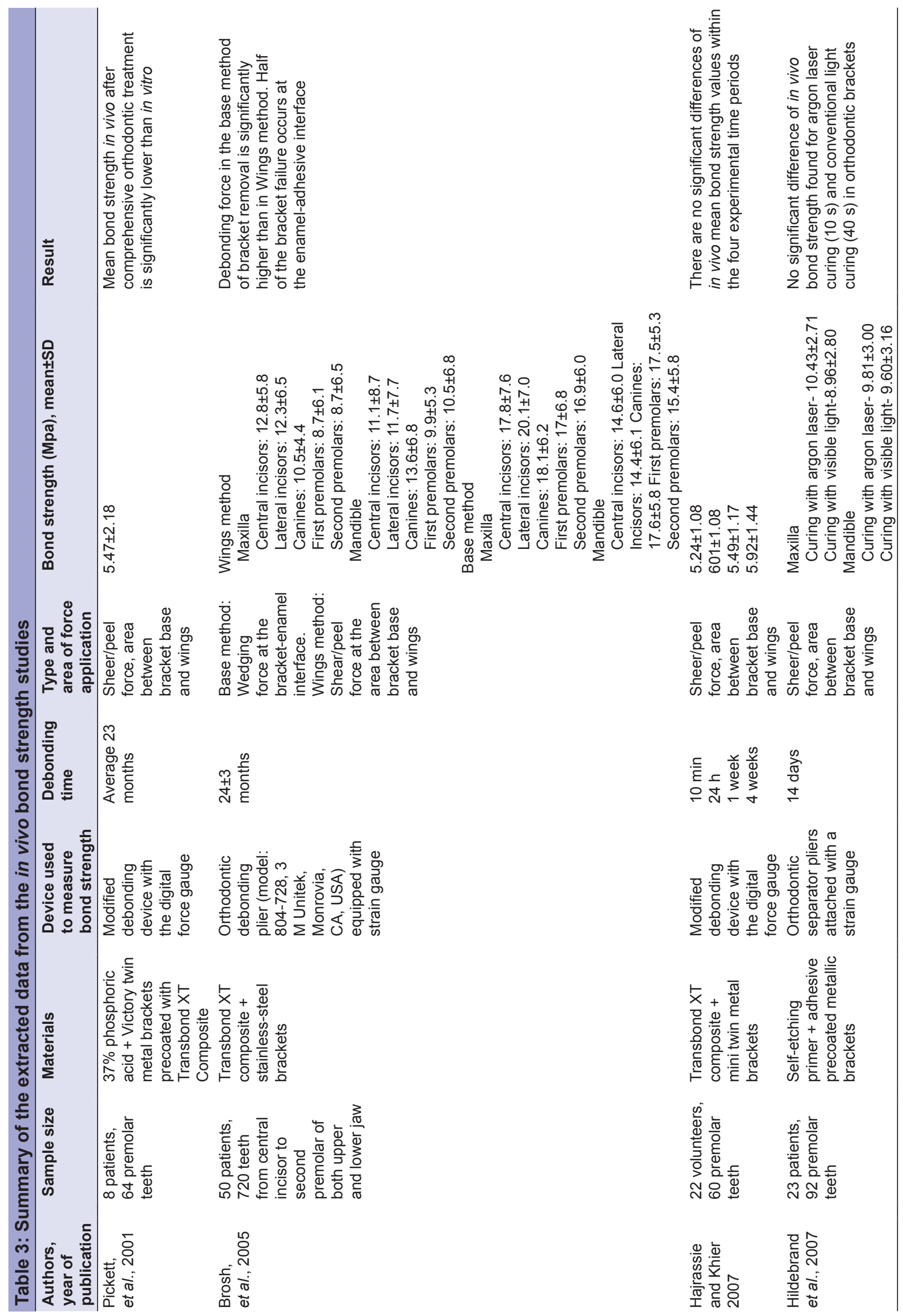




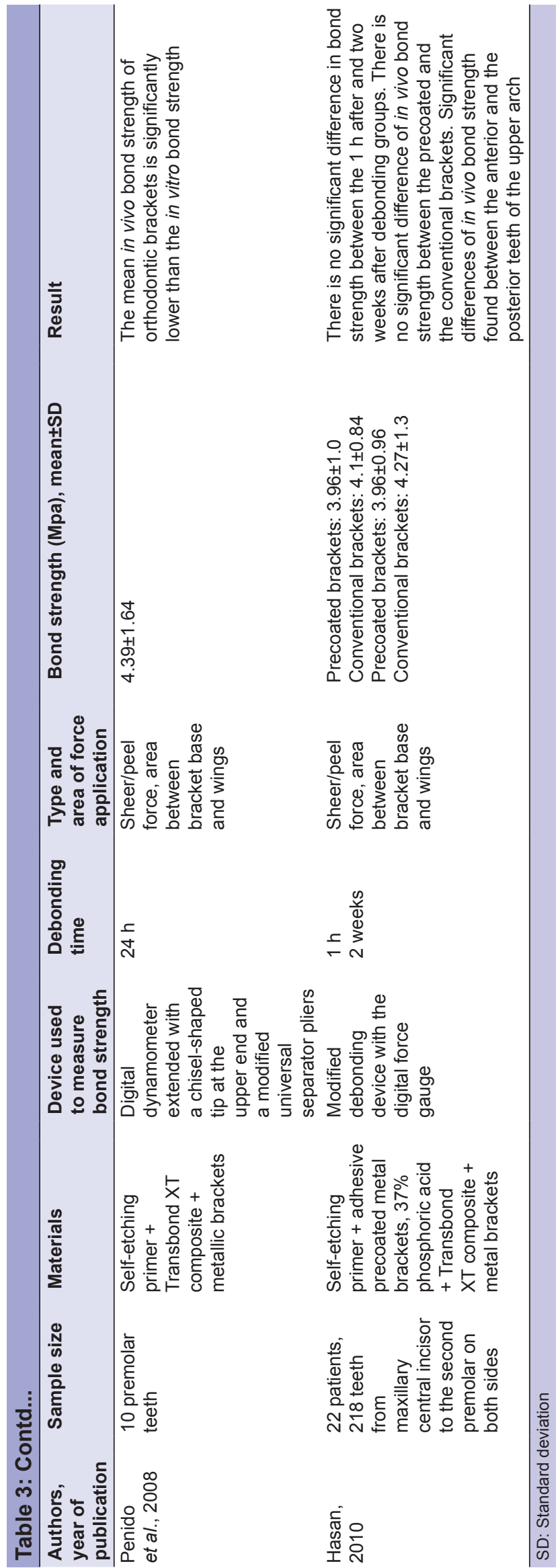

resin is found to be the most popular orthodontic adhesive in the majority of the studies. ${ }^{[4,5-7]}$ Therefore, it was not possible to determine the efficacy of different adhesives in vivo other than composites. This necessitates further in vivo studies to evaluate the bonding efficiency of resin-modified glass ionomers and other fluoride releasing orthodontic adhesives clinically which are used in regular practice. Only conventional and adhesive precoated metallic brackets were experimented to measure the bond strength. ${ }^{[2-7]}$ One study measured and compare the bond strength of both conventional and adhesive precoated metallic brackets and found no difference ${ }^{[6]}$ Although this may encourage the clinician to use adhesive precoated metallic brackets because of the simplicity and less chair-side time, despite that the adhesive precoated brackets exhibited the lower bond strength than the conventional metallic brackets in both ex vivo and in vitro studies. ${ }^{[8,9]}$ But in both studies, the mean bond strength of the adhesive precoated brackets was 10.31 and $5.4 \mathrm{MPa}$, respectively, which is justifiable for the clinical situation. ${ }^{[2]}$ Still, no study is conducted to measure the bond strength of ceramic brackets in vivo inspite of the fact that difference of bond strength found between the metallic and the ceramic brackets in vitro. ${ }^{[10,11]}$ Polymerization of the orthodontic adhesives by the visible light source for $40 \mathrm{~s}$ in vivo was the most popular method..$^{[2,4,6,7]}$ As an alternative to the visible light, curing of orthodontic adhesives also experimented with argon laser in both in vivo and in vitro and the xenon arc light source in vitro, respectively. ${ }^{[7,12,13]}$ In comparison to conventional curing, the only advantage is that the curing time is reduced in both methods without affecting the bond strength and thus can be a good alternative clinically for a reduced chair-side time.

After 10 min of bonding brackets, there was no difference of bond strength in vivo at further time intervals up to 4 weeks. This also favors the findings from an in vitro study that the bond strength of orthodontic adhesive can increase up to first $10 \mathrm{~min}$ of bonding and after that, it reaches a state of equilibrium. ${ }^{[14]}$ Like most of the in vitro and ex vivo studies, bond strength is measured traditionally in the units of $\mathrm{N} / \mathrm{m}^{2}$ or Mpa after dividing the debonding force by the bracket surface area, assuming that the stress developed at the point of bracket failure is uniformly distributed over the entire bracket base. Although it may be useful for comparing the results from the previous studies such calculations are not reliable because according to the finite element analysis, the stress distribution within the adhesive 
layer, in the brackets, and enamel during debonding is inhomogeneous. ${ }^{[15]}$ Moreover, the approximation of the actual contact area of the bracket base, variation of the bracket surface morphology, and adhesive thickness are the impediments for converting the units of debonding force to stress. Therefore, the peak debonding force developed at the site of failure should be the measure of the bonding efficiency.

In vivo bond strength was first successfully, measured using digital force gauge attached to a stainless-steel crossmember and a modified-elastic spacer instrument. ${ }^{[2]}$ The instrument was designed to apply shear/peel load on the area between the base and the bracket base. The short arm of the crossmember was made similar, to the debonding plier at the tip for engaging the bracket in the space between the base and the wings in the mesiodistal direction. The elastic spacer instrument was soldered to metal pads at the tip with a groove allowing free movement of the crossmember during force application. Before debonding, the occlusal splints were given to patients for protection as one pad of the plier tip was rested on the occlusal surface of the teeth and the other pad on the force gauge with the crossmember engaged to the bracket. While the plier handles were compressed, the plier pads move away from each other and applied force on the occlusal surface of the tooth on one side and the force gauge on the other side. This allowed shearing of the bracket by the crossmember engaged to it, while, the force gauge recorded the peak force at the time of bond failure. Later, this idea of measuring bond strength was followed by three more studies, but with little modification. ${ }^{[4-6]}$ Instead of inserting the acrylic splint into the mouth during debonding, rubber pad was applied on the tip of the modified elastic spacer instrument. ${ }^{[5]}$ As the digital force gauge is capable of measuring both tension and compression, in two studies, they encased the force gauge in an aluminum box snugly to measure the true shear force without any external disturbances. ${ }^{[4,6]}$ But according to the finite element analysis, brackets are exposed to combinations of tension, shear peel, and torsional loading modes in all directions clinically during debonding. ${ }^{[16]}$

Debonding force and the resulting iatrogenic enamel damage during debonding orthodontic brackets may vary with the type of force, the area of force application, and the type of debonding instrument used. ${ }^{[16-18]}$ The bracket failure at the interface between the adhesive and the enamel removes a layer of enamel. ${ }^{[19]}$ Only, the manufacturer-made bracket debonding pliers are capable of performing consistent separation at the bracket-adhesive interface without any damage to the enamel. ${ }^{[17]}$ One study found to measure the bond strength of orthodontic brackets using regular debonding plier. ${ }^{[3]}$ They applied the load on two different locations on the bracket-wedging force on the area between the bracket base and enamel (base method) and shear/peel force on the area between the wings and base of the bracket (wings method). The later area and the mode of force application were also preferred by most of the in vivo bond strength studies. ${ }^{[2,4-7]}$ The greater debonding force was required in the base method than in the wings method because further the point of force application is away from the bracket/adhesive/enamel interface, the lesser force will be required to dislodge the brackets. Despite that, in both methods, the majority of bracket debonding occurred at the adhesive/enamel interface suggesting the imminent enamel damage. Besides, structural damage to the bracket is more common in wings method. No study found to evaluate the effect of tensile loading modes on the bond strength in vivo. Although the tensile debonding test may not simulate the bracket debonding in clinical situations, it can provide valuable information on the relative bond strengths for the comparison of debonding techniques, clinically. However, in comparison to the sheer/peel loading, the force required for debonding brackets lesser when a tensile load is applied on the bracket found in a finite element analysis. ${ }^{[16]}$

Several studies used a strain gauge to measure the bracket debonding force in vivo. ${ }^{[3,7]}$ Strain gauges are the electrical transducers that sense the mechanical deformation and converts it into a range of electrical resistance ${ }^{[2]}$ Strain gauges are accurate and lightweight but very much responsive to the temperature change which can influence to alter the measurements. In both of the studies, no measures were mentioned for compensating the temperature alteration which may have an influence on the bond strength results.

Validation and interexaminer calibration is very important to assess the accuracy of any experimental instrument. Although the two studies validated their debonding device by comparing the results with the gold standard universal testing machine, ${ }^{[2,4]}$ no study calibrates their experimental instrument for interexaminer reliability.

In the majority of the studies, the in vivo bond strength of orthodontic brackets on premolar teeth was considered only. ${ }^{[2,4,5,7]}$ Two studies measured the bond strength of orthodontic brackets on different teeth in vivo under 
different experimental conditions. ${ }^{[3,6]}$ In one study, in vivo bond strength of orthodontic brackets was measured on different teeth from central incisor to the second premolar in both upper and lower jaw to detect the difference in bond strength between the base method and the Wing method of bracket removal in similar teeth and the teeth in the same quadrant, while the other study performed in vivo bond strength measurement only on the upper arch from the central incisor to the second premolar and found that the bond strength of orthodontic brackets are higher on the anterior teeth segment than on the posterior. Similar outcomes were also seen in several in vitro studies, measuring the bond strength of orthodontic brackets on different teeth types. ${ }^{[20-22]}$ This warrants further investigation, particularly in vivo to produce an established data on the debonding force of orthodontic brackets on specific tooth groups in both the upper and lower jaw.

The limitation of this review is that only six full-text articles could be analyzed and the meta-analysis could not be performed due to the lack of homogenous study results.

\section{CONCLUSION}

- In vivo bond strength tests are assumed to be the most valid because all the parameters of the oral environment and their effect on the in vivo aging of the bracket bonding system is considered

- In comparison to the conventional laboratory-based in vitro studies still, furthermore progress to be made in this study field

- The evaluation can be done by measuring the peak debonding force developed during the bracket failure rather than the controversial and the conventional idea of dividing the debonding force by the total bracket surface area to calculate the average stress on the bracket

- An identical standardized method should be adapted using similar and validated debonding device to carry out the comprehensive research studies, for evaluating and comparing the bonding performance of a wide range of orthodontic adhesives and brackets under different experimental conditions inside the oral environment.

Financial support and sponsorship

USM Fellowship 2017/18.

\section{Conflicts of interest}

There are no conflicts of interest.

\section{REFERENCES}

1. Downs SH, Black N. The feasibility of creating a checklist for the assessment of the methodological quality both of randomised and non-randomised studies of health care interventions. J Epidemiol Community Health 1998;52:377-84.

2. Pickett KL, Sadowsky PL, Jacobson A, Lacefield W. Orthodontic in vivo bond strength: Comparison with in vitro results. Angle Orthod 2001;71:141-8.

3. Brosh T, Kaufman A, Balabanovsky A, Vardimon AD. In vivo debonding strength and enamel damage in two orthodontic debonding methods. J Biomech 2005;38:1107-13.

4. Hajrassie MK, Khier SE. In vivo and in vitro comparison of bond strengths of orthodontic brackets bonded to enamel and debonded at various times. Am J Orthod Dentofacial Orthop 2007;131:384-90.

5. de Oliveira Penido SM, de Sousa Resende Penido CV, dos Santos-Pinto A, Sakima T, Fontana CR. In vivo and in vitro study, with or without thermocycling, of shear bond strength of brackets bonded with halogen light. Rev Dent Press Ortod Ortop Facial 2008;13:66-76.

6. Hassan AH. Shear bond strength of precoated orthodontic brackets: An in vivo study. Clin Cosmet Investig Dent 2010;2:41-5.

7. Hildebrand NK, Raboud DW, Heo G, Nelson AE, Major PW. Argon laser vs conventional visible light-cured orthodontic bracket bonding: An in vivo and in vitro study. Am J Orthod Dentofacial Orthop 2007;131:530-6.

8. Bishara SE, Olsen M, Von Wald L. Comparisons of shear bond strength of precoated and uncoated brackets. Am J Orthod Dentofacial Orthop 1997;112:617-21.

9. Cal-Neto JP, Miguel JA, Zanella E. Effect of a self-etching primer on shear bond strength of adhesive precoated brackets in vivo. Angle Orthod 2006;76:127-31.

10. Habibi M, Nik TH, Hooshmand T. Comparison of debonding characteristics of metal and ceramic orthodontic brackets to enamel: An in vitro study. Am J Orthod Dentofacial Orthop 2007;132:675-9.

11. Eslamian L, Borzabadi-Farahani A, Mousavi N, Ghasemi A. A comparative study of shear bond strength between metal and ceramic brackets and artificially aged composite restorations using different surface treatments. Eur J Orthod 2012;34:610-7.

12. Talbot TQ, Blankenau RJ, Zobitz ME, Weaver AL, Lohse CM, Rebellato J, et al. Effect of argon laser irradiation on shear bond strength of orthodontic brackets: An in vitro study. Am J Orthod Dentofacial Orthop 2000;118:274-9.

13. Sfondrini MF, Cacciafesta V, Pistorio A, Sfondrini G. Effects of conventional and high-intensity light-curing on enamel shear bond strength of composite resin and resin-modified glass-ionomer. Am J Orthod Dentofacial Orthop 2001;119:30-5.

14. Chamda RA, Stein E. Time-related bond strengths of light-cured and chemically cured bonding systems: An in vitro study. Am J Orthod Dentofacial Orthop 1996;110:378-82.

15. Eliades T, Brantley WA. The inappropriateness of conventional orthodontic bond strength assessment protocols. Eur J Orthod 2000;22:13-23.

16. Katona TR. A comparison of the stresses developed in tension, shear peel, and torsion strength testing of direct bonded orthodontic brackets. Am J Orthod Dentofacial Orthop 1997;112:244-51.

17. Zarrinnia K, Eid NM, Kehoe MJ. The effect of different debonding techniques on the enamel surface: An in vitro qualitative study. Am J Orthod Dentofacial Orthop 1995;108:284-93.

18. Klocke A, Kahl-Nieke B. Influence of force location in orthodontic shear bond strength testing. Dent Mater 2005;21:391-6.

19. Janiszewska-Olszowska J, Tandecka K, Szatkiewicz T, Sporniak-Tutak K, Grocholewicz K. Three-dimensional quantitative analysis of adhesive remnants and enamel loss resulting from debonding orthodontic molar tubes. Head Face Med 2014;10:37.

20. Linklater RA, Gordon PH. An ex vivo study to investigate bond strengths of different tooth types. J Orthod 2001;28:59-65.

21. Hobson RS, McCabe JF, Hogg SD. Bond strength to surface enamel for different tooth types. Dent Mater 2001;17:184-9.

22. Oztürk B, Malkoç S, Koyutürk AE, Catalbas B, Ozer F. Influence of different tooth types on the bond strength of two orthodontic adhesive systems. Eur J Orthod 2008;30:407-12. 